\title{
O.S.P.
}

L'orientation scolaire et professionnelle

$33 / 2$ | 2004

Adolescences - 1

\section{Vygotski. Conscience, inconscient, émotions}

Paris : La Dispute

\section{Katia Kostulski}

\section{(2) OpenEdition}

Journals

Édition électronique

URL : https://journals.openedition.org/osp/2188

DOI : 10.4000/osp.2188

ISSN : 2104-3795

Éditeur

Institut national d'étude du travail et d'orientation professionnelle (INETOP)

Édition imprimée

Date de publication : 15 juin 2004

Pagination : 339-340

ISSN : 0249-6739

Référence électronique

Katia Kostulski, «L. Vygotski. Conscience, inconscient, émotions », L'orientation scolaire et professionnelle [En ligne], 33/2 | 2004, mis en ligne le 10 novembre 2009, consulté le 21 septembre 2021. URL : http:// journals.openedition.org/osp/2188; DOI : https://doi.org/10.4000/osp.2188

Ce document a été généré automatiquement le 21 septembre 2021.

(c) Tous droits réservés 


\section{Vygotski. Conscience, inconscient, émotions}

Paris : La Dispute

Katia Kostulski

\section{RÉFÉRENCE}

Préface de Yves Clot

Paris : La Dispute

1 "Conscience, inconscient, émotions", réunit trois textes de Vygotski parus entre 1925 et 1932. Ceux-ci - d'une étonnante actualité - traitent de questions majeures en psychologie : le problème de la conscience, celui de la vie psychique et de l'inconscient, et enfin celui du développement des émotions. On ne peut donc que se féliciter de l'initiative d'une telle publication. Cela d'autant plus que l'ouvrage n'en est pas moins original: sa longue préface - que signe Yves Clot - constitue en effet un important développement des thèses vygotskiennes. C'est pourquoi après une présentation succincte des problèmes ouverts dans ces trois textes, nous reviendrons sur quelquesunes des perspectives proposées dans cette préface.

2 «La question de la nature psychologique de la conscience est laissée de côté dans notre littérature scientifique de manière opiniâtre et délibérée ». Voilà le constat qui initie $L a$ conscience comme problème de la Psychologie du comportement. Dans ce texte, Vygotski développe, au plan des théories et des méthodes de la psychologie scientifique de l'époque, les causes et les conséquences d'une telle omission. Le grain d'observation du comportement permet-il d'élucider les phénomènes de conscience? Les méthodes utilisées, fondées sur les réactions observables, s'avèrent «inconsistantes " pour l'étudier, de sorte que ce qui chez l'humain oriente et organise le comportement échappe à la psychologie. C'est alors le comportement qui perd sa composante psychologique. Il n'est ainsi pas étonnant, dans ce cadre, que le comportement de l'animal et celui de l'homme soient relégués au même rang, précipitant alors la 
psychologie dans une subordination au physiologique: «La biologie engloutit la sociologie et la physiologie engloutit la psychologie ». Pourtant, avance l'auteur, la conscience est une composante inaliénable du comportement: "l'homme pense toujours dans son for intérieur; cela ne manque jamais d'influer sur son comportement ». Plus encore, observe-t-il, « la conscience est un problème de structure du comportement ». Étudier le comportement, c'est étudier sa structure, son contenu, ses formes, son histoire. C'est dans les rapports du comportement à l'expérience du sujet que Vygotski élabore une définition de la conscience humaine : la conscience est l'expérience vécue d'expériences vécues. Naît alors la possibilité de distinguer les formes de conscience animale de la conscience humaine, par la variabilité des champs d'expériences de l'homme: l'expérience sociale, le langage. La proposition de l'auteur ne manque pas d'audace : la conscience humaine serait « en quelque sorte » un rapport social à soi-même.

3 Psychisme, conscience, inconscient, désignent selon Vygotski des questions « touchant aux principes de constitution de la science psychologique elle-même». C'est pourquoi il examine dans ce texte la façon dont les courants de la psychologie s'expliquent ces trois mots. Il reprend pour cela différents courants de son époque du point de vue des rapports qu'ils construisent avec ces trois termes, telles la réflexologie, la phénomènologie, la psychanalyse, et en dégage trois systèmes psychologiques très différents fondés, respectivement, sur le refus d'étudier le psychisme, l'étude du psychisme par l'intermédiaire du psychique, et la connaissance du psychisme par l'inconscient. Chacun de ces courants, avance l'auteur, mène à sa manière à une impasse, notamment dans la façon dont ils posent les rapports entre le psychique et le physiologique. Pour Vygotski, le psychisme n'est pas un processus à part, en dehors, au-dessus ou complémentaire, il est une caractéristique qualitative des fonctions supérieures du cerveau. Il en déduit la nécessité de développer une psychologie dialectique qui, sans confondre les processus physiques et psychiques, reconnaisse que le psychologique est l'unité dialectique du physique et du psychique. C'est de ce nouveau point de vue que l'auteur examinera les trois termes dans la suite du texte. Le psychisme est-il la conscience? La conscience est-elle un processus linéaire ? La vie psychique est-elle continue? Que déduire dès lors sur l'inconscient, du constat omniprésent en psychologie: certains éléments de la vie psychique, qui ont cette particularité d'être dissociés des mots, continuent d'influer sur le comportement alors qu'ils sont hors du champ de conscience?

4 Enfin, dans Les émotions et leur développement chez l'enfant, Vygotski ne cherche pas tant à décrire le développement des émotions qu'à affirmer l'idée même d'un développement de ces émotions, à identifier leur siège et leur origine somatique ou psychique, pour enfin examiner sur cette base la façon dont les processus émotionnels peuvent prendre une place dans le fonctionnement psychologique. Sommes-nous affligés parce que nous pleurons ou pleurons-nous parce que nous sommes affligés? Les réactions somatiques sont-elles spécifiques à des émotions particulières? Les émotions peuvent-elles exister indépendamment de réactions végétatives? Ces réactions végétatives sont-elles causes, conséquences ou simplement parties des émotions? Vygotski examine ces questions en reprenant l'histoire des théories des émotions en psychologie expérimentale, et en discutant les théories du développement phylogénétique et ontogénétique des émotions. L'auteur en déduit le caractère nomade des émotions dans la vie psychologique: les émotions n'ont pas «de place fixée à tout jamais ». Un état émotionnel se transforme en un autre, un affect s'insère dans n'importe quelle 
structure à laquelle il est lié, les émotions non résolues continuent d'exister. La psychopathologie constitue dès lors le champ dans lequel le statut des émotions et le processus de leur développement dans la vie psychologique peut être défini. La névrose apparait comme un modèle de vie psychique «bouleversée par suite de perturbation de l'activité émotionnelle». La psychose révèle une modification pathologique des rapports entre les processus intellectuels et émotionnels. Dans la pensée réaliste, les émotions accompagnent les processus intellectuels dans la pensée. Dans la pensée autistique, c'est tout le système de pensée qui est structurellement désorganisé. Les émotions ont perdu la place structurelle qu'elles occupaient auparavant : les idées sont gouvernées, non par les processus intellectuels, mais par des tendances émotionnelles. La pensée est alors subordonnée à la logique du sentiment.

5 Le texte d'Yves Clot, Vygotski: la conscience comme liaison, enfin, n'est une préface que dans sa première partie. Il y présente les trois textes qui constituent l'ouvrage au sein de l'œuvre dense - 180 textes connus - de Vygotski. La seconde partie de la contribution justifie le choix de ces trois textes. En effet, Yves Clot y prolonge et renouvelle une réflexion sur la conscience dont ces textes constituent les fondations. L'auteur poursuit le dialogue initié par Vygotski avec la psychanalyse, en reprenant les développements plus récents des théories psychanalytiques, ouvrant ainsi des perspectives nouvelles et riches sur une conceptualisation de la vie psychique. La conscience apparaît ici fondamentalement comme un rapport réel de transformation de l'expérience ou d'échec de cette transformation. Yves Clot ouvre une alternative à la conceptualisation freudienne de l'inconscient, cet "inaccompli qui insiste», qui est défini ici comme « une réalisation en gésine, une activité en mal de liaison ». Deux idées fondamentales sont avancées sur cette base par Yves Clot. D'abord, les conflits inconscients pourraient avoir d'autres sources que les conflits de l'enfance : «le sexuel infantile n'a pas le monopole du pulsionnel ». Ensuite, réinterrogeant la place du social dans la vie psychique du sujet, l'auteur avance que le social, loin d'être à côté ou audehors du sujet, « est aussi là quand le sujet est seul». Plus encore, agir sur le social serait peut-être le moyen le plus sûr d'agir indirectement sur la vie subjective. 